\title{
Hysteresis and Return-Point Memory in Deterministic Cellular Automata
}

\author{
Jürgen Goicoechea and Jordi Ortín \\ Departament d'Estructura i Constituents de la Matèria, Facultat de Física, Universitat de Barcelona, \\ Diagonal 647, 08028 Barcelona, Catalonia, Spain \\ (Received 2 August 1993; revised manuscript received 1 November 1993)
}

\begin{abstract}
We have investigated hysteresis and the return-point memory (RPM) property in deterministic cellular automata with avalanche dynamics. The RPM property reflects a partial ordering of metastable states, preserved by the dynamics. Recently, Sethna et al. [Phys. Rev. Lett. 70, 3347 (1993)] proved this behavior for a homogeneously driven system with static disorder. This Letter shows that the partial ordering and the RPM can be displayed as well by systems driven heterogeneously, as a result of its own evolution dynamics. In particular, we prove the RPM property for a deterministic 2D sandpile automaton driven at a central site.
\end{abstract}

PACS numbers: $64.60 . \mathrm{My}, 05.40 .+\mathrm{j}, 75.60 . \mathrm{Ej}$

Hysteresis is a macroscopic evidence of energy dissipation in externally driven physical systems. We are familiar, for example, with the hysteresis cycle displayed by the magnetization of a ferromagnet when subjected to an external magnetic field. In many systems the hysteresis trajectories have been found experimentally to be highly reproducible from cycle to cycle-though sometimes only after a number of training cycles. This, in spite of the fact that a detailed examination of the trajectories reveals, instead of a smooth evolution, a sequence of sharp discontinuities separated by intervals of null activity, is signature of an evolution by avalanches [1].

Moreover, the hysteretic behavior exhibits many times the striking property of return point memory (RPM). It has been observed in a variety of physical systems: ferromagnets [2], solids undergoing structural [3] and spin [4] transitions, charge-density waves [5], adsorption of gases by porous solids [6], etc. Very recently Sethna et al. [7] have shown that a random-field Ising model (RFIM) at zero temperature displays the RPM property, as a consequence of the fact that (i) there is an ordered sequence of metastable states available to the system, and (ii) in the absence of thermal fluctuations, this sequence is preserved by the dynamics.

In this Letter we will show that a dynamically preserved ordered sequence of metastable states (and hence the RPM property) can arise as well quite naturally in systems with homogeneous properties, as a result of its own evolution dynamics.

As a paradigm of this new kind of system with RPM, we have considered the fully deterministic cellular automaton introduced by Wiesenfeld, Theiler, and $\mathrm{McNa}$ mara (WTM) in the context of self-organized criticality [8]; this cellular automaton evolves in a self-organized way into a critical state, in which avalanches of all sizes can occur (limited only by the size of the system itself) and the amplitude distribution of avalanches follows a power law [9].

The WTM sandpile automaton that we have studied to investigate the RPM property consists of an $N \times N$ square lattice with an integer-value function $z(x, y)$ defined on each lattice site. $N$ is odd to provide for a unique central site. A configuration of the automaton is given by the ensemble $\{z(x, y)\}$ for all lattice sites. In order to study hysteresis we must provide dynamic rules, in discrete time, for the forward and for the reverse evolution of the automaton. These are given by central seeding/removal,

$$
z(0,0) \rightarrow z(0,0) \pm 1,
$$

and forward/reverse flow:

$$
\text { If } z(x, y) \gtrless \pm z^{*} \Rightarrow\left\{\begin{array}{l}
z(x, y) \rightarrow z(x, y) \mp 4, \\
z(x, y+1) \rightarrow z(x, y+1) \pm 1, \\
z(x, y-1) \rightarrow z(x, y-1) \pm 1, \\
z(x+1, y) \rightarrow z(x+1, y) \pm 1, \\
z(x-1, y) \rightarrow z(x-1, y) \pm 1 .
\end{array}\right.
$$

$z^{*}$ is a constant threshold, for which we have taken the value $z^{*}=4$ as in Ref. [8]. The evolution in the forward direction is given by the upper signs and the upper inequality. Seeding of the central site provides the driving force for the automaton to evolve. The rules are applied in the following way: (1) is iterated until $z(0,0)>z^{*}$; then (2) is repeatedly applied synchronously (i.e., all sites are updated simultaneously) until $z(x, y) \leq z^{*}$ in all lattice sites. At this moment the system has reached a new metastable configuration. Further forward evolution is achieved by repeating the whole procedure again. In addition, we have chosen the following:

$z(x, y)=0$ for all $x, y$ (initial conditions),

$z(x, y)=0$ on the boundary (boundary conditions).

Our choice of the initial conditions and the fact that the evolution rules are the same in all sites make the properties of the system homogeneous.

To evolve in the reverse direction we have considered the lower signs and the lower inequality. The rules are applied in exactly the same way as before. That this is the natural choice of rules for the reverse evolution follows from the observation that, if we were to choose (3) and (4) as initial and boundary conditions for the reverse evolution, this set of rules would give rise to the same se- 


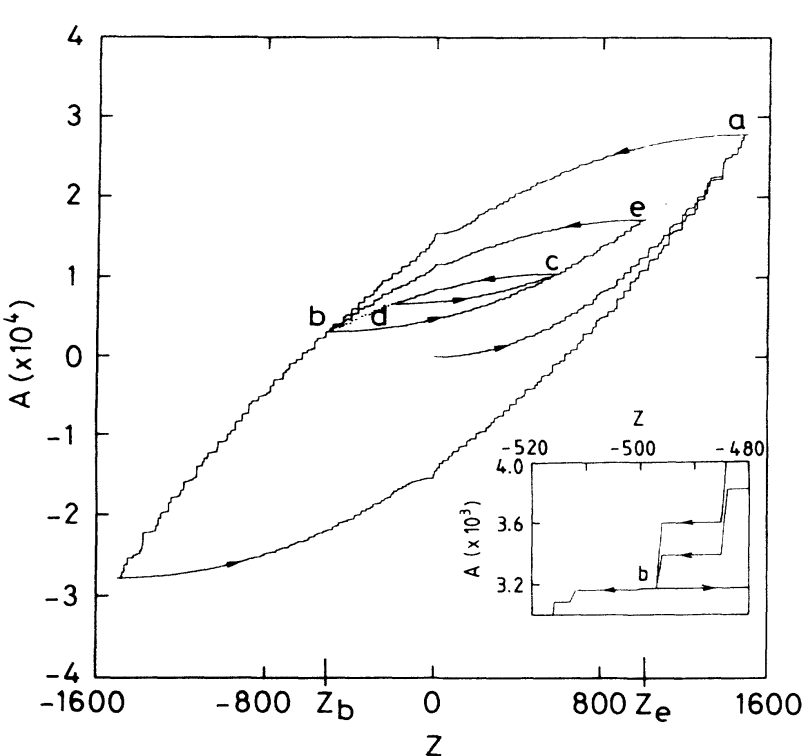

FIG. I. Automaton trajectories showing hysteresis and return-point memory. We have plotted $A$ (number of times that some lattice site becomes critical) as a function of $Z$ (external driving force) for a $2 \mathrm{D}$ lattice of size $23 \times 23$. Notice the RPM property: In the internal loop between $b$ and $e$, the reverse path starting at $\mathbf{e}$ rejoins the outer path exactly at $\mathbf{b}$, the state previously left. The inset shows the neighborhood of the turning point $\mathbf{b}$ in detail. The broken lines represent the trajectories followed when $Z$ is not reversed at e and d, respectively, and provide new examples of RPM.

quence of configurations than in the forward evolution, except for the sign of the integer variable $z(x, y)$. In other words, the reverse sandpile appears now as the mirror image across the plane $z(x, y)=0$ of the forward sandpile. In addition, the mirror symmetry guarantees that the backward evolution of the cellular automaton leads also to a SOC state [9].

Let us define macroscopic variables to describe the dynamic evolution of the system. Since the automaton is externally driven by central seeding and central removal [rule (1)] we define a variable $Z$ that is initially 0 , and $Z \rightarrow Z \pm 1$ every time that rule (1) is applied. This variable measures the external driving force applied to the automaton. The outcome of driving the automaton is the occurrence of avalanches of varying sizes. We define a variable $A$, conjugate to $Z$, to measure the number of times that any lattice site becomes critical; i.e., $A$ is initially 0 , and $A \rightarrow A \pm 1$ every time that rules (2) are applied.

The evolution of $A$ as a function of $Z$ is shown in Fig. 1. Initially the automaton is at $Z=0, A=0$; a monotonic increase of $Z$ leads to the evolution given by the ascending jerky line. When state $\mathbf{a}$ is reached we stop central seeding and begin central removal: The reverse evolution goes above the previous forward one, displaying hysteresis. The internal trajectories illustrate the RPM property: If the driving force $Z$ is increased from $Z_{b}$ to

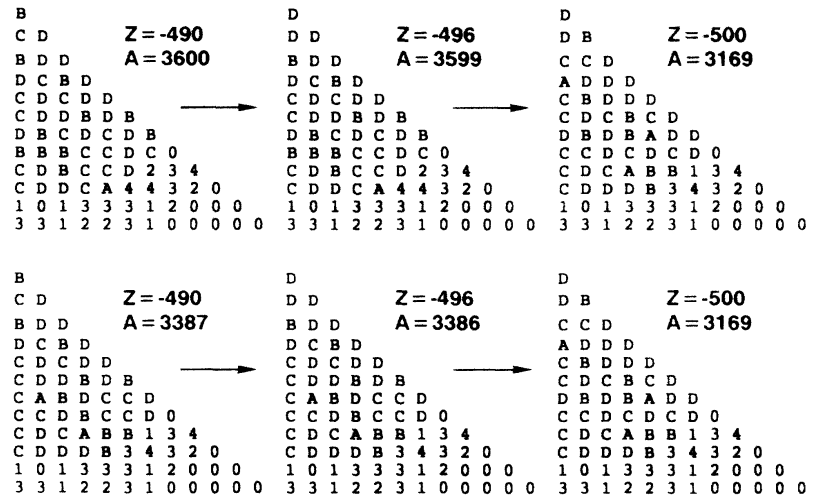

FIG. 2. Automaton microstructures. Displayed are two sequences of states at three different values of $Z$ for the two descending trajectories in the inset of Fig. 1. The upper sequence corresponds to the outer trajectory and the lower one to the inner trajectory. Representation of negative numbers: $\mathbf{A}$ for -1 , B for $-2, \mathbf{C}$ for -3 , and $\mathbf{D}$ for -4 . Because of the symmetry of the WTM dynamics it is not necessary to display the whole lattice; the upper site of each state is the central site.

$Z_{e}$, when it comes back to $Z_{b}$ the automaton returns precisely to the same state $\mathbf{b}$ from which it left the outer trajectory (i.e., the internal trajectory remembers state b). The inset in Fig. 1 shows in detail the neighborhood of state $\mathbf{b}$ : The trajectory of increasing $Z$ leaves $\mathbf{b}$ with zero slope, because there is a dead interval before $Z$ exceeds threshold in some lattice site; the trajectory of decreasing $Z$ in the partial loop rejoins the state $b$ exactly, and at this point there is a discontinuity in slope that reflects a discontinuous increase in the number of lattice sites with $z(x, y)$ values falling below threshold. The same RPM property is found for smaller loops within this loop (such as the one formed between $\mathbf{c}$ and $\mathbf{d}$ ), and so on: The state of the automaton depends on an entire hierarchy of previous turning points. The broken lines, finally, represent the trajectories obtained if $Z$ was not reversed at point $\mathbf{d}$ and at point e, which again display RPM. Figure 2 presents two examples of the evolution of the microstructure; they correspond to the two descending trajectories shown in the inset of Fig. 1, leading exactly to the same state $b$.

To understand the origin of RPM in the WTM automaton, we work out now an alternative description of its dynamics based on new sets of local variables.

(i) Partial ordering of states.-Instead of using $\{z(x, y)\}$, we characterize a state of the automaton by a new ensemble $\{a(x, y)\}$ such that the new state variables $a(x, y)$ evolve (increase or decrease) monotonously with the external driving force $Z$. A good candidate for $a(x, y)$ is the number of times that the lattice site $(x, y)$ has become critical, as given by

$a(x, y)=0$ for all $x, y$ (initial conditions),

$a(x, y)=0$ on the boundary (boundary conditions), 


$$
\text { if } z(x, y) \gtrless \pm z^{*} \Rightarrow a(x, y) \rightarrow a(x, y) \pm 1 \text { (dynamics). }
$$

It is worth noting that $a(x, y)$ can take any integer value in the interval $(-\infty, \infty)$.

There is now a natural partial ordering of the states: a state $\mathrm{s}=\left\{a_{s}(x, y)\right\} \geq \mathrm{r}=\left\{a_{r}(x, y)\right\}$ if $a_{s}(x, y) \geq a_{r}(x, y)$ for each site $(x, y)$ in the system. It should remain clear that this ordering is only partial, and many pairs of states that the automaton displays do not have a definite ordering relationship.

(ii) The partial ordering is preserved by the $d y$ namics. - This property is actually a restatement of Middleton's no passing rule [10] in a different context. Consider a state $s(t)$ that evolves under an external driving $Z_{s}(t)$, and another state $\mathbf{r}(t)$ that evolves under $Z_{r}(t)$. Suppose that initially they are ordered, in such a way that $\mathrm{s}(0) \geq \mathrm{r}(0)$. Then, as long as $Z_{s}(t) \geq Z_{r}(t)$ at all times $t$, the ordering $\mathrm{s}(t) \geq \mathrm{r}(t)$ remains valid.

To prove this property we have to define on each lattice site of the automaton a function that follows the evolution of $z(x, y)$ due only to external contributions:

$$
F(x, y)=z(x, y)+q a(x, y) .
$$

$q$ is the number of units lost or gained by the variable $z(x, y)$ when flow takes place; in our case $q=4$. $F(x, y)$ measures the local driving on each lattice site. In general, the interest of introducing $F(x, y)$ is that the local state variable $a(x, y)$ follows a monotonic evolution under the local field $F(x, y)$, independent of the previous history of $F(x, y)$. For the particular system studied here, the initial conditions and the evolution rules have been chosen to be the same in all lattice sites and therefore, in addition, the relationship between $a(x, y)$ and $F(x, y)$ (Fig. 3 ) is independent of the site $(x, y)$ considered.

Next, recalling the definition of $F(x, y)$, we note that $F$ can also be written in terms of $a$ and the external driving force $Z$ alone, in the form

$$
\begin{aligned}
& F(0,0)=\sum_{\langle u, v\rangle} a(u, v)+Z, \\
& F(x, y)=\sum_{\langle u, v\rangle} a(u, v) \text { if }(x, y) \neq(0,0) .
\end{aligned}
$$

Here $\langle u, v\rangle$ indicates summation over the $q=4$ nearest neighbors of the site $(x, y)$. These two equations are easily derived from the definition of $F(x, y)$ and the observation that the external contributions to the change of $z(x, y)$ come from (i) its $q$ nearest neighbors [every time that one of them becomes critical, as measured by $a(u, v)]$ and (ii) the external driving force $Z$ if the site considered is the central site.

Equations (9) and (10) are in a sense analogous to the expression of the internal field experienced by a magnetic spin in a two-dimensional Ising model, with $Z$ playing the role of an external magnetic field applied to the central site only. Hence we can follow the argument of Ref. [7]

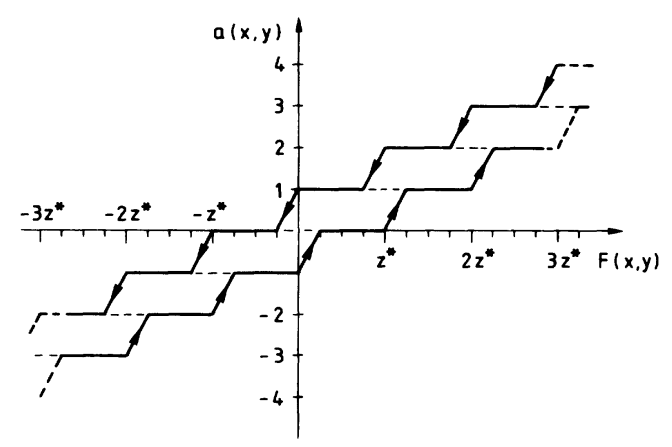

FIG. 3. Local evolution. Shown is the evolution of the local state variable $a(x, y)$ under the local field $F(x, y)$ for an arbitrary lattice site $(x, y)$. The ordering of states is preserved by the dynamics of the cellular automaton because there is a unique $F(x, y)-a(x, y)$ relationship for each one of the two directions of evolution.

to prove that the ordering $\mathbf{s}(t) \geq \mathbf{r}(t)$ is preserved: If it is not preserved, there must be a first time $t$ such that $a_{r}(t)>a_{s}(t)$ on some lattice site $(x, y)$; since the evolution of $a$ with $f$ is monotonic, at that time $F_{r}(x, y)$ must be larger than $F_{s}(x, y)$; but, according to (9) and (10), this is not possible since all the nearest neighbors in $\mathbf{s}$ have $a(u, v)$ larger than or equal to the corresponding ones in $r$, and $Z_{s}(t) \geq Z_{r}(t)$.

In addition, it is worth noting that the partial ordering would not be preserved if the automaton was seeded in a different lattice site, randomly chosen at each time step. Random seeding would play here the same role as thermal fluctuations in the RFIM.

(iii) The dynamics are adiabatic. - By this we mean that, starting in some state $r$, any monotonic change of the external driving from $Z_{r}$ to $Z_{s}$ will make the state evolve in the same way and into the same final state s. In other words, the evolution is not affected by the rate at which $Z$ changes. This behavior follows naturally from the rules defining the dynamics of the cellular automaton.

Once established, these three properties (partial ordering, no passing, adiabaticity) are sufficient to prove the RPM property (see Ref. [7]).

Return-point memory.-Suppose a state s(0) evolves under an external driving $Z(t)$, where $Z(0) \leq Z(t)$ $\leq Z(T)$ for $0<t<T$, with $Z(t)$ not necessarily monotonic. Then the final state of the system depends only on $Z(T)$, and is independent of the time $T$ or the history $Z(t)$. In particular, a system coming back to a previous value of driving will return exactly to the same state, provided that the driving remains within these bounds.

To conclude our analysis, we use Eqs. (8), (9), and (10) to show that the variables $z(x, y)$ and $a(x, y)$ are related by

$$
\begin{aligned}
& z(0,0)=\sum_{\langle u, v\rangle} a(u, v)-q a(0,0)+Z \\
& z(x, y)=\sum_{\langle u, v\rangle} a(u, v)-q a(x, y) \text { if }(x, y) \neq(0,0) .
\end{aligned}
$$


According to these expressions $a(x, y)$ satisfies a discrete Poisson equation, in which $z(x, y)$ [and $z(0,0)-Z$ in the central site] play the role of sources. Hence a metastable configuration $\{z(x, y)\}$ is uniquely determined from $\{a(x, y)\}$, and vice versa, if $Z$ is known.

The formalism used here to analyze the RPM property is actually of wider application. It suffices to identify $a(x, y)$, the local response, and $F(x, y)$, the local driving. Then, our analysis proves that any deterministic cellular automata will present an ordered sequence of states preserved by the dynamics (and hence will display RPM), if there is a unique relationship between $a(x, y)$ and $F(x, y)$ which can be different from site to site but must be monotonic and independent of the previous history of $F(x, y)$ [11].

The requirements for RPM can be satisfied by deterministic models in two different ways. (i) In models driven homogeneously, a dynamically preserved ordered sequence is only achieved if static disorder is introduced - for instance, in the initial conditions. The RFIM at $T=0$, driven by an external magnetic field [12], and the WTM automaton driven homogeneously from a random initial configuration, are examples of models in this category. (ii) In models with heterogeneous driving the ordered sequence of metastable states may arise naturally as a consequence of their own evolution dynamics. There is no need of static disorder: Both the initial conditions and the system properties are homogeneous. In this paper we have studied in detail the WTM automaton with central driving, and have outlined the general procedure to study other cellular automata in this second category.

The two different mechanisms might be simultaneously operative in real physical systems: The initial effect of a weak frozen disorder could be amplified by the intrinsic dynamics of the system. This assumption would explain why systems with relatively low concentrations of frozenin heterogeneities could nevertheless exhibit RPM.

This work has received financial support from the CICyT (Spain), Project No. MAT 92-884. We would like to thank A. Planes, E. Vives, and P. A. Lindgård for helpful discussions, and J. P. Sethna and S. Kartha for making their paper available as a preprint. The authors acknowledge the Centre de Supercomputació de Catalunya (CESCA) for use of their computing facilities.
[1] In magnetic materials the discontinuities give rise to the Barkhausen effect; see, for example, P. J. Cote and L. V. Meisel, Phys. Rev. Lett. 67, 1334 (1991). In materials undergoing a martensitic transformation, to a trail of acoustic emission pulses; see, for example, A. Planes, J. L. Macqueron, M. Morin, and G. Guénin, Phys. Status Solidi (a) 66, 717 (1981).

[2] D. C. Jiles and D. L. Atherton, J. Appl. Phys. 55, 2115 (1984); J. A. Barker, D. E. Schreiber, B. G. Huth, and D. H. Everett, Proc. R. Soc. London A 386, 251 (1983).

[3] In the martensitic transformation of metallic alloys: J. Ortín, J. Appl. Phys. 71, 1454 (1992), and references therein. In ammonium chloride: Smits et al., Z. Phys. Chem. A 166, 97 (1933); 175, 359 (1936); Z. Phys. Chem. B 41, 215 (1938).

[4] E. W. Müller, H. Spiering, and P. Gütlich, J. Chem. Phys. 79, 1439 (1983).

[5] Z. Z. Wang and N. P. Ong, Phys. Rev. B 34, 5967 (1986).

[6] J. Katz, J. Phys. Chem. 53, 1166 (1949).

[7] J. P. Sethna, K. Dahmen, S. Kartha, J. A. Krumhansl, B. W. Roberts, and J. D. Shore, Phys. Rev. Lett. 70, 3347 (1993).

[8] K. Wiesenfeld, J. Theiler, and B. McNamara, Phys. Rev. Lett. 65, 949 (1990).

[9] The concept of self-organized criticality (SOC) is due to P. Bak, C. Tang, and K. Wiesenfeld, Phys. Rev. Lett. 59, 381 (1987); Phys. Rev. A 38, 364 (1988).

[10] A. A. Middleton, Phys. Rev. Lett. 68, 670 (1992).

[11] One might think that the cellular automaton version of the Burridge-Knopoff model for earthquakes, introduced by Z. Olami, H. J. S. Feder, and K. Christensen [Phys. Rev. Lett. 68, 1244 (1992)], would be another example with homogeneous driving; it turns out that this model (no matter its degree of conservation or anisotropy) cannot exhibit RPM, because the ordering of states is not preserved by the dynamics. The reason is that the redistribution of strain to the neighbors is proportional to the strain in the relaxing site. Since a site $(x, y)$ can relax from different values of strain (above threshold), there is not a unique relationship between $a(x, y)$ and $F(x, y)$ : The final value of $a(x, y)$ reached after a relaxation depends on the past history of $F(x, y)$. This makes the no passing rule to fail for sufficiently large excursions of the external driving.

[12] The RFIM at $T=0$, driven by an external field, can also be viewed as a deterministic cellular automaton with spatial randomness in the initial conditions and homogeneous driving. 